\title{
Modeling of Subharmonics and Chaos in DC Motor Drives
}

\author{
K.T. Chau, J.H. Chen, C.C. Chan, and David T.W. Chan* \\ Department of Electrical \& Electronic Engineering, The University of Hong Kong, Pokfulam, Hong Kong \\ *Department of Electrical \& Communications Engineering, Hong Kong Technical College, Tsing Yi, Hong Kong
}

\begin{abstract}
In this paper, the nonlinear dynamics of both voltage-mode and current-mode controlled de motor drive systems are presented. The investigation is based on the derivation of the discrete mappings that describe their system subharmonics and chaos in the continuous conduction mode of operation. It illustrates that different bifurcation diagrams can be obtained by using different modes of control while varying the same system parameters. A unified modeling approach for the period-1 and hence the period-p orbits as well as their stability analysis during both voltage-mode and current-mode of control is proposed and verified.
\end{abstract}

\section{INTRODUCTION}

The belief in our common sense that simple systems exhibiting simple behavior is not always true. In fact, the investigation of some simple switching dc-dc converters has revealed their remarkable complex and chaotic behaviors [1][5].

The investigation onto chaotic behavior in power electronics circuits was launched in late 1980's. Since linear system theory is ill-suited to investigate the subharmonics and chaotic phenomena occurred in power electronics circuits, the most attractive approach has been the iterative nonlinear mapping. Recently, the two-dimensional mapping has been manipulated to investigate the chaotic behavior of voltage-mode or current-mode controlled dc-dc converters [4],[5]. However, these studies have been focused on the use of linearization such as the constant or compensating ramp current reference in current-mode control. Notice that this current reference is practically variable, depending on the output voltage variation.

On the other hand, the investigation onto chaotic behavior of industrial motor drives is becoming more and more attractive [6]-[9]. The chaotic behavior of PWM inverter-fed induction motor drives has been investigated by using numerical analysis [6]. It has also been attempted to investigate the chaotic behavior of brushless dc motor drives by ignoring the switching effect and approximately transforming into the Lorenz system [7]. Recently, the nonlinear dynamics and chaotic behavior of industrial dc motor drives have been investigated numerically and analytically, without ignoring the switching effect or accepting rough assumptions [8],[9].

It is the purpose of this paper to systematically investigate the nonlinear dynamics of both voltage-mode and currentmode controlled dc motor drives. Based on a dc chopper-fed permanent-magnet (PM) dc motor drive, a unified analytical modeling approach for investigating their subharmonics and chaos as well as their stability analysis will been presented. Computer simulations will be carried out to study these subharmonics and chaotic behavior. Finally, theoretical results will be verified by comparing with those obtained by PSpice simulation.

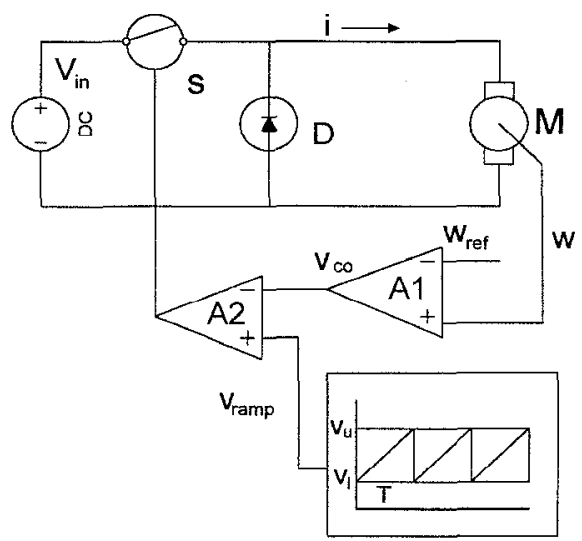

Fig. 1. Voltage-mode controlled de motor drive.

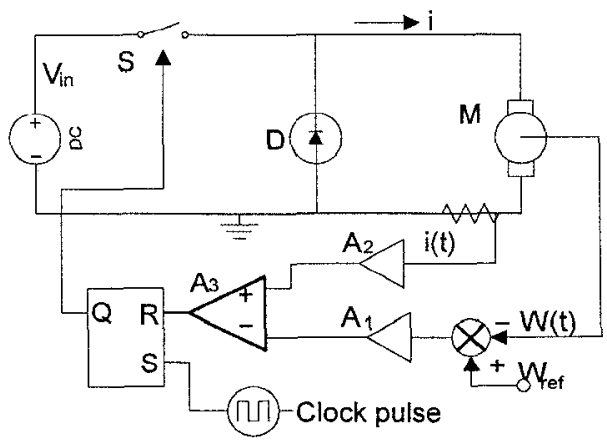

Fig. 2. Current-mode controlled de motor drive.

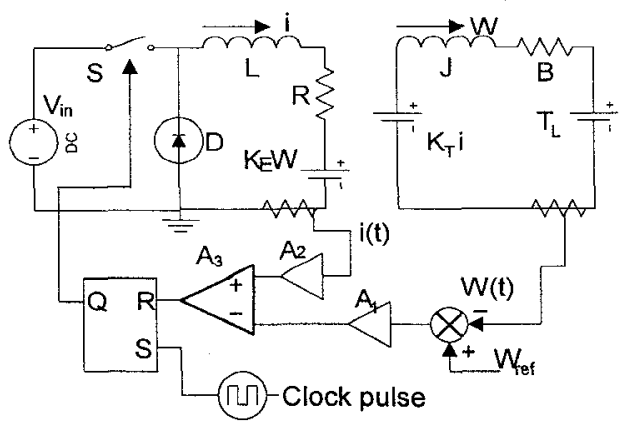

Fig. 3. Equivalent circuit of current-mode controlled dc motor drive. 


\section{MODELING OF DRIVE SYSTEMS}

As shown in Figs. 1 and 2, the voltage-mode and currentmode buck-type dc chopper-fed PM dc motor drives operating in the continuous conduction mode are used for exemplification. The equivalent circuit of the current-mode controlled dc motor drive is shown in Fig. 3.

Whatever the dc drive system is controlled by voltagemode or current-mode, it always operates with two topological stages during the continuous conduction mode. Stage 1 is that power switch S is on and diode D is off, and stage 2 is that $S$ is off and $D$ is on. Therefore, the system equation can be divided into two linear differential equations as given by:

- Stage 1 ( $\mathrm{S}$ is on and D is off):

$$
\frac{d}{d t}\left(\begin{array}{c}
\omega(t) \\
i(t)
\end{array}\right)=\left(\begin{array}{cc}
-B / J & K_{T} / J \\
-K_{E} / L & -R / L
\end{array}\right)\left(\begin{array}{c}
\omega(t) \\
i(t)
\end{array}\right)+\left(\begin{array}{c}
-T_{l} / J \\
V_{i n} / L
\end{array}\right)
$$

- Stage 2 ( $\mathrm{S}$ is off and D is on):

$$
\frac{d}{d t}\left(\begin{array}{c}
\omega(t) \\
i(t)
\end{array}\right)=\left(\begin{array}{cc}
-B / J & K_{T} / J \\
-K_{E} / L & -R / L
\end{array}\right)\left(\begin{array}{c}
\omega(t) \\
i(t)
\end{array}\right)+\left(\begin{array}{c}
-T_{l} / J \\
0
\end{array}\right)
$$

where $\omega(t)$ is the instantaneous speed, $i(t)$ instantaneous armature current, $R$ armature resistance, $L$ armature inductance, $V_{\text {in }}$ dc supply voltage, $K_{E}$ back-EMF constant, $K_{T}$ torque constant, $B$ viscous damping, $J$ load inertia, and $T_{l}$ load torque.

By defining the state vector $\mathbf{X}(t)$ and the following matrices $\mathbf{A}, \mathbf{E}_{\text {on }}, \mathbf{E}_{\text {off }}$ as:

$$
\begin{aligned}
& \mathbf{A}=\left(\begin{array}{cc}
-B / J & K_{T} / J \\
-K_{E} / L & -R / L
\end{array}\right), \quad \mathbf{X}(t)=\left(\begin{array}{c}
\omega(t) \\
i(t)
\end{array}\right) \\
& \mathbf{E}_{\text {on }}=\left(\begin{array}{c}
-T_{l} / J \\
V_{\text {in }} / L
\end{array}\right), \quad \mathbf{E}_{\text {off }}=\left(\begin{array}{c}
-T_{l} / J \\
0
\end{array}\right)
\end{aligned}
$$

the system equation given by (1) and (2) can then be rewritten as:

$$
\dot{\mathbf{X}}(t)=\mathbf{A} \mathbf{X}(t)+\mathbf{E}_{k} \quad(k=\text { on, off })
$$

Since $k$ is topology dependent, hence time dependent, the system equation given by (5) is in fact a time-varying state equation. Thus, this closed-loop drive system is a secondorder non-autonomous dynamical system. The drive system switches from one stage to the other one whenever the control signal $v_{c}(t)$ is equal to the carrier signal $v_{r}(t)$.

For a voltage-mode drive system, the control signal $v_{c}(t)$ can be expressed as:

$v_{c}(t)=g\left(\omega(t)-\omega_{r e f}\right)$

where $\omega_{\text {ref }}$ is the reference speed, and $g$ feedback gain. The carrier signal ramp voltage $v_{r}(t)$ is represented by:

$v_{r}(t)=v_{l}+\left(v_{u}-v_{l}\right) t / T$

where $v_{l}$ and $v_{u}$ are respectively the lower and upper voltages of the ramp signal, and $T$ is its period.

For a current-mode drive system, the control signal $v_{c}(t)$ can be expressed as: $v_{c}(t)=g_{i} i(t)-g_{\omega}\left(\omega_{\text {ref }}-\omega(t)\right)$

where $g_{i}$ and $g_{\omega}$ are armature current and voltage feedback gains, respectively. The carrier signal in the interval of clock pulses is zero.

Therefore, the unified condition of both voltage-mode and current-mode drive systems can be represented as:

$\mathbf{E}_{3}\left(\mathbf{X}(t)-\mathbf{E}_{4}\right)=v_{r}(t)$

where $\mathbf{E}_{3}=(g, 0)$ and $v_{r}(t)=v_{l}+\left(v_{u^{-}}-v_{l}\right) t / \mathrm{T}$ for the voltage-mode, $\mathbf{E}_{3}=(g, 1)$ with $g=g_{\omega} / g_{i}$ and $v_{r}(t)=0$ for the current-mode, and $\mathbf{E}_{4}=\left(\omega_{\text {ref }}, 0\right)$.

\section{MODELING OF SUBHARMONICS AND CHAOS}

Given the initial conditions $\mathbf{X}\left(t_{0}\right)$, the analytical solution of the system equation given by (5) can be expressed as:

$$
\begin{aligned}
\mathbf{X}(t) & =\Phi\left(t-t_{0}\right) \mathbf{X}\left(t_{0}\right)+\int_{t_{0}} \Phi(t-\tau) \mathbf{E}_{k} d \tau \\
& =-\mathbf{A}^{-1} \mathbf{E}_{k}+\Phi\left(t-t_{0}\right)\left(\mathbf{X}\left(t_{0}\right)+\mathbf{A}^{-1} \mathbf{E}_{k}\right)
\end{aligned}
$$

where $\Phi(t)=e^{\mathbf{A} t}$ is so-called the state transition matrix. $\mathbf{E}_{k}$ is $\mathbf{E}_{\text {on }}$ or $\mathbf{E}_{\text {off. }}$. The corresponding discrete state vector is denoted as $\mathbf{X}_{n}=\mathbf{X}(n T)$. The iterative function that maps this vector to its successive one $\mathbf{X}_{n+1}=\mathbf{X}((n+1) T)$ is called the Poincaré map $P: \mathfrak{R}^{2} \rightarrow \mathfrak{R}^{2}$ which is defined as:

$\mathbf{X}_{n+1}=P\left(\mathbf{X}_{n}\right)$

\section{A. The generalized Poincaré Map for Voltage-Mode Drives}

Since the voltage-mode $\mathrm{dc}$ drive system operates periodically with $T, \mathbf{X}(t)$ is generally sampled at the beginning of every ramp cycle. Within each $T$, there are two possible situations - a skipped cycle because of the absence of intersection between $v_{c}$ and $v_{r}$, and an intersected cycle in which there is at least one intersection between $v_{c}$ and $v_{r}$.

For the skipped cycle, $\mathrm{S}$ does not change its state, remaining either on or off. The corresponding Poincaré map $P$ can be easily derived from (10) as given by:

$\mathbf{X}_{n+1}=-\mathbf{A}^{-1} \mathbf{E}_{k}+\Phi(T)\left(\mathbf{X}_{n}+\mathbf{A}^{-1} \mathbf{E}_{k}\right) \quad(k=$ on, off $)$

For the intersected cycle, S may change $m$ times when $v_{c}$ crosses $v_{r}$ by $m_{v}$ times within the same $T$, so-called multiple pulsing. The corresponding intersections occur at:

$t=n T+\delta_{i} T, \quad 0=\delta_{0}<\delta_{1}<, \cdots,<\delta_{m}<1 \quad\left(i=1, \cdots, m_{v}\right)(13)$ Hence, the generalized Poincaré map can be expressed as the following iterative form:

$$
\left\{\begin{array}{r}
\mathbf{X}\left(n T+\delta_{i} T\right)=-\mathbf{A}^{-1} \mathbf{E}_{k}+\Phi\left(\delta_{i} T-\delta_{i-1} T\right)\left(\mathbf{X}\left(n T+\delta_{i-1} T\right)+\mathbf{A}^{-1} \mathbf{E}_{k}\right) \\
h\left(\delta_{i}\right)=\mathbf{E}_{3}\left(\mathbf{X}\left(n T+\delta_{i} T\right)-\mathbf{E}_{4}\right)-v_{r}\left(\delta_{i} T\right)=0 \\
i=1,2, \cdots, m_{v}
\end{array}\right.
$$

$$
\mathbf{X}_{n+1}=-\mathbf{A}^{-1} \mathbf{E}_{k}+\Phi\left(T-\delta_{m} T\right)\left(\mathbf{X}\left(n T+\delta_{m} T\right)+\mathbf{A}^{-1} \mathbf{E}_{k}\right)
$$

where $k$ is off or on, depending on whether $v_{c}$ is over or under $v_{r}$. 


\section{B. The generalized Poincaré Map for Current-Mode Drives}

As shown in Fig. 3, the interval of closing or opening a switch may be longer than a clock cycle under certain conditions. Thus, $\mathbf{X}(t)$ is generally sampled at the beginning of the clock pulse that makes $S$ changing from off to on, instead of every clock cycle. Hence, the drive system always operates in stage 1 first and then in stage 2 for each sampling interval. By defining the intervals of stages 1 and 2 as $\delta_{o n} T$ and $\delta_{o f f} T$, respectively, the interval of the Poincare map becomes $m_{i} T=\left(\delta_{o n}+\delta_{o f f}\right) T$. Thus, $\mathbf{X}\left(\delta_{o n} T\right)$ and $\mathbf{X}_{n+1}$ can be directly derived from (10) as given by:

$$
\begin{aligned}
& \left\{\begin{array}{c}
\mathbf{X}\left(\delta_{o n} T\right)=-\mathbf{A}^{-1} \mathbf{E}_{o n}+\Phi\left(\delta_{o n} T\right)\left(\mathbf{X}_{n}+\mathbf{A}^{-1} \mathbf{E}_{o n}\right) \\
h\left(\delta_{o n}\right)=\mathbf{E}_{3}\left(\mathbf{X}\left(\delta_{o n} T\right)-\mathbf{E}_{4}\right)=0
\end{array}\right. \\
& \mathbf{X}_{n+1}=-\mathbf{A}^{-1} \mathbf{E}_{o f f}+\Phi\left(m_{i} T-\delta_{o n} T\right)\left(\mathbf{X}\left(\delta_{o n} T\right)+\mathbf{A}^{-1} \mathbf{E}_{o f f}\right)
\end{aligned}
$$

On the other hand, $m_{i}$ can be obtained as the minimum integer which is larger than $\delta_{o n}$ and fulfills $h\left(m_{i}\right)<0$. Hence, the Poincare map can be written as:

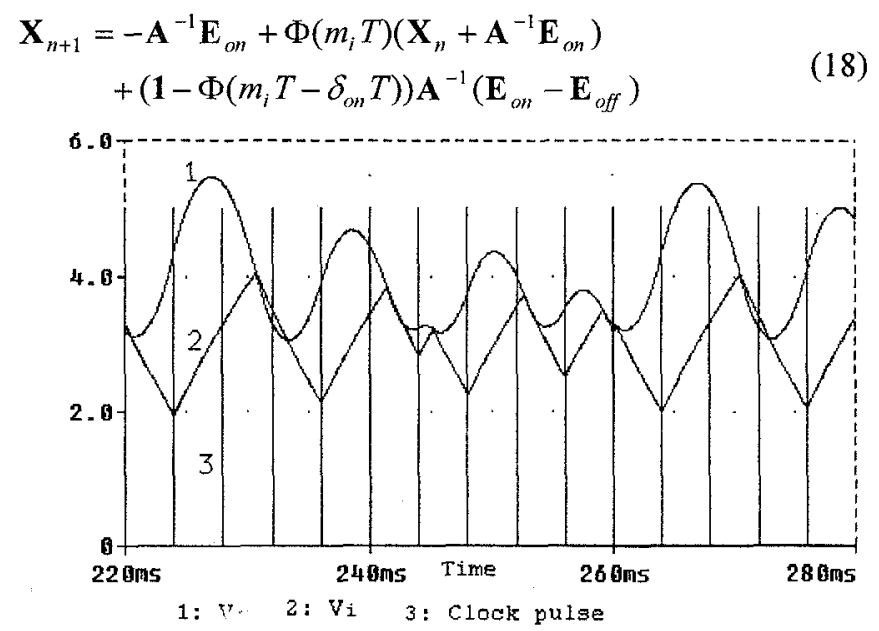

Fig. 4. Chaotic waveforms of $v_{\omega}$ and $v_{i}$ against clock pulse.

\section{Modeling of Periodic Orbits}

The above generalized Poincare map of the voltage-mode dc drive system is so general that it includes cycle skipping and multiple pulsing. Due to their presence, it is inconvenient to analyze the steady-state periodic orbits and their stability. Also, the presence of multiple pulsing can greatly increase the switching losses, which should be avoided by using a latch or sample-and-hold. Therefore, instead of using the generalized one, the detailed analysis of periodic orbits will adopt a specific Poincaré map that the orbits cross the ramp signal once per cycle - mathematically, $m_{v}=1$.

The steady-state periodic solution of the dc drive system can be a fixed point, so-called the period- 1 orbit $\mathbf{X}^{*}$, or a cycle point, so-called the period- $p$ orbit $\left\{\mathbf{X}_{1}^{*}, \cdots, \mathbf{X}_{p}^{*}\right\} \quad(p>1)$. The corresponding specific Poincaré maps are described as:
$\mathbf{X}^{*}=P\left(\mathbf{X}^{*}\right)$

$\mathbf{X}_{k+1}^{*}=P\left(\mathbf{X}_{k}^{*}\right) \quad(k=1, \cdots, p-1), \quad \mathbf{X}_{1}^{*}=P\left(\mathbf{X}_{p}^{*}\right)$

Firstly, the period- 1 orbit is analyzed. Since $m=1$ and the orbit must start with $v_{l}<v_{c}<v_{u}$, the drive system operates in stage 2 whose interval is $\delta_{o f f} T$ and then in stage 1 whose interval is $\delta_{o n} T$. By defining the duty cycle $\delta=\delta_{o f f}$, the corresponding Poincaré map can be obtained as:

$$
\begin{aligned}
\mathbf{X}_{n+1}= & -\mathbf{A}^{-1} \mathbf{E}_{o f f}+\Phi(T)\left(\mathbf{X}_{n}+\mathbf{A}^{-1} \mathbf{E}_{o f f}\right) \\
& +(\mathbf{1}-\Phi(T-\delta T)) \mathbf{A}^{-1}\left(\mathbf{E}_{o f f}-\mathbf{E}_{o n}\right)
\end{aligned}
$$

Substituting (21) into the map given by (19), the period-1 orbit can be obtained as:

$$
\left\{\begin{aligned}
\mathbf{X}^{*}= & -\mathbf{A}^{-1} \mathbf{E}_{\text {off }}+(\mathbf{1}-\Phi(T))^{-1}(\mathbf{1}-\Phi(T-\delta T)) \mathbf{A}^{-1} \\
& \times\left(\mathbf{E}_{\text {off }}-\mathbf{E}_{\text {on }}\right) \\
h(\delta)= & \mathbf{E}_{3}\left[-\mathbf{A}^{-1} \mathbf{E}_{\text {off }}+\Phi(\delta T)\left(\mathbf{X}^{*}+\mathbf{A}^{-1} \mathbf{E}_{\text {off }}\right)-\mathbf{E}_{4}\right]-v_{r}(\delta T) \\
= & 0
\end{aligned}\right.
$$

Hence, provided that $\delta \in(0,1), \mathbf{X}^{*}$ can be obtained from (22). Notice that $\mathrm{X}^{*}$ is the period-1 orbit if and only if $v_{c}$ always lies between $v_{l}$ and $v_{u}$. Its characteristic multipliers $\left(\lambda_{1}, \lambda_{2}\right)$ are the eigenvalues of the Jacobian matrix of that mapping (21), which is given by:

$$
D P\left(\mathbf{X}^{*}\right)=\Phi(T)+\Phi(T-\delta T)\left(\mathbf{E}_{o f f}-\mathbf{E}_{o n}\right) T \frac{\partial \delta}{\partial \mathbf{X}^{*}}
$$

where $\frac{\partial \delta}{\partial \mathbf{X}^{*}}=\left(\frac{\partial \delta}{\partial \omega}, \frac{\partial \delta}{\partial i}\right)$. According to the implicit-function theorems, $\frac{\partial \delta}{\partial \mathbf{X}^{*}}$ can be deduced from (22) as:

$$
\begin{aligned}
\frac{\partial \delta}{\partial \mathbf{X}^{*}} & =-\left(\frac{\partial h}{\partial \delta}\right)^{-1} \frac{\partial h}{\partial \mathbf{X}^{*}} \\
& =-\left[\mathbf{E}_{3} \Phi(\delta T)\left(\mathbf{A} \mathbf{X}^{*}+\mathbf{E}_{\text {off }}\right) T-\left(v_{u}-v_{l}\right)\right]^{-1} \mathbf{E}_{3} \Phi(\delta T)
\end{aligned}
$$

It is known that the fixed point of the mapping is stable if and only if its characteristic multipliers all lie within the unit circle in the complex plane. If one of their magnitudes equals unity, this fixed point can be a bifurcation point.

Secondly, the period $-p$ orbit $\left\{\mathbf{X}_{1}^{*}, \cdots, \mathbf{X}_{p}^{*}\right\}$ is analyzed. By defining $\delta_{1}, \cdots, \delta_{p}$ as the duty cycles of $p$ periods with $p>1$, it indicates that $v_{c}(t)$ crosses the voltage ramp $p$ times at $\left(n T+\delta_{1} T\right), \cdots,\left((n+p) T+\delta_{p} T\right)$, respectively. Based on the specific Poincaré map derived in (21), the $p$-fold iterative Poincaré map can be formulated as:

$$
\begin{aligned}
\mathbf{X}_{n+p} & =P^{(p)}\left(\mathbf{X}_{n}\right) \\
& =-\mathbf{A}^{-1} \mathbf{E}_{o f f}+\Phi(p T)\left(\mathbf{X}_{n}+\mathbf{A}^{-1} \mathbf{E}_{o f f}\right) \\
& +\sum_{j=1}^{p}\left[\Phi((p-j) T)\left(\mathbf{1}-\Phi\left(T-\delta_{j} T\right)\right)\right] \mathbf{A}^{-1}\left(\mathbf{E}_{o f f}-\mathbf{E}_{o n}\right)
\end{aligned}
$$

By using (20), the period- $p$ orbit can then be derived as: 


$$
\begin{aligned}
\mathbf{X}_{1}^{*}= & -\mathbf{A}^{-1} \mathbf{E}_{o f f}+(\mathbf{1}-\Phi(p T))^{-1} \sum_{j=1}^{p}[\Phi((p-j) T) \\
& \left.\times\left(\mathbf{1}-\Phi\left(T-\delta_{j} T\right)\right)\right] \mathbf{A}^{-1}\left(\mathbf{E}_{o f f}-\mathbf{E}_{o n}\right) \\
\mathbf{X}_{i}^{*}= & -\mathbf{A}^{-1} \mathbf{E}_{o f f}+\Phi((i-1) T)\left(\mathbf{X}_{1}^{*}+\mathbf{A}^{-1} \mathbf{E}_{o f f}\right) \\
& -\sum_{j=1}^{i-1}\left[\Phi((i-j-1) T)\left(\mathbf{1}-\Phi\left(T-\delta_{j} T\right)\right)\right] \\
& \times \mathbf{A}^{-1}\left(\mathbf{E}_{o f f}-\mathbf{E}_{o n}\right) \quad(i=2, \cdots, p) \\
h_{i}(\mathbf{d})= & \mathbf{E}_{3}\left[-\mathbf{A}^{-1} \mathbf{E}_{o f f}+\Phi\left(\delta_{i} T\right)\left(\mathbf{X}_{i}^{*}+\mathbf{A}^{-1} \mathbf{E}_{o f f}\right)-\mathbf{E}_{4}\right] \\
& -v_{r}\left(\delta_{i} T\right)=0 \quad(i=1, \cdots, p)
\end{aligned}
$$

where $\Phi(0)=1$ and $\mathbf{d}=\left(\delta_{1}, \cdots, \delta_{p}\right)$. By substituting (26) and (27) into (28), d can be determined. Provided that $\delta_{i} \in(0,1)$ for $i=1, \cdots, p, \mathbf{X}_{1}^{*}, \cdots, \mathbf{X}_{p}^{*}$ can then be obtained from (26) and (27). If the condition $v_{l}<v_{c}<v_{u}$ is guaranteed in each period, $\left\{\mathbf{X}_{1}^{*}, \cdots, \mathbf{X}_{p}^{*}\right\}$ is a period- $p$ orbit.

Due to the cyclic property of a cycle point, $\left\{\mathbf{X}_{2}^{*}, \cdots, \mathbf{X}_{p}^{*}, \mathbf{X}_{1}^{*}\right\}, \cdots,\left\{\mathbf{X}_{p}^{*}, \mathbf{X}_{1}^{*}, \cdots, \mathbf{X}_{p-1}^{*}\right\}$ are also period- $p$ orbits which correspond to the same subharmonic frequency. If $\mathbf{X}_{1}^{*}=\cdots=\mathbf{X}_{p}^{*}$, the period- $p$ orbit becomes the period-1 orbit, which shows that period-1 is its subset.

For the period- $p$ orbits $\left\{\mathbf{X}_{1}^{*}, \cdots, \mathbf{X}_{p}^{*}\right\}, \mathbf{X}_{1}^{*}$ is a fixed point of the $p$-fold iterative Poincaré map. Therefore, its characteristic multipliers $\left(\lambda_{1}, \lambda_{2}\right)$ are the eigenvalues of the Jacobian matrix of that mapping, which is given by:

$$
\begin{aligned}
D P^{(p)}\left(\mathbf{X}_{\mathfrak{l}}^{*}\right)= & \Phi(p T)+\sum_{j=1}^{p}[\Phi((p-j) T) \\
& \left.\times \Phi\left(T-\delta_{j} T\right)\left(\mathbf{E}_{\text {off }}-\mathbf{E}_{o n}\right) T \frac{\partial \delta_{j}}{\partial \mathbf{X}_{l}^{*}}\right]
\end{aligned}
$$

By substituting (28) into (29), it results:

$\mathbf{H}\left(\mathbf{d}, \mathbf{X}_{1}^{*}\right)=\left[h_{1}\left(\mathbf{d}, \mathbf{X}_{1}^{*}\right), \cdots, h_{p}\left(\mathbf{d}, \mathbf{X}_{1}^{*}\right)\right]^{T}=\mathbf{0}$

Hence, according to the implicit-function theorems, the partial derivative in (32) can be expressed as:

$$
\begin{aligned}
\frac{\partial \mathbf{d}}{\partial \mathbf{X}_{1}^{*}}= & \left(\frac{\partial \delta_{1}}{\partial \mathbf{X}_{1}^{*}}, \cdots, \frac{\partial \delta_{p}}{\partial \mathbf{X}_{1}^{*}}\right)^{T}=-\left[\frac{\partial \mathbf{H}}{\partial \mathbf{d}}\right]^{-1}\left[\frac{\partial \mathbf{H}}{\partial \mathbf{X}_{1}^{*}}\right] \\
\frac{\partial \mathbf{H}}{\partial \mathbf{d}}= & {\left[\frac{\partial h_{i}}{\partial \delta_{j}}\right]_{p \times p} } \\
= & \operatorname{diag}\left[\mathbf{E}_{3} \Phi\left(\delta_{i} T\right)\left(\mathbf{A} \mathbf{X}_{i}^{*}+\mathbf{E}_{o f f}\right) T-\left(v_{t u}-v_{l}\right)\right] \\
& +\left[\mathbf{E}_{3} \Phi\left(\delta_{i} T\right) \Phi\left((i-j) T-\delta_{j} T\right)\left(\mathbf{E}_{o f f}-\mathbf{E}_{o n}\right) T\right]_{p \times p} \\
\frac{\partial \mathbf{H}}{\partial \mathbf{X}_{1}^{*}} & =\left[\frac{\partial h_{1}}{\partial \mathbf{X}_{1}^{*}}, \cdots, \frac{\partial h_{p}}{\partial \mathbf{X}_{1}^{*}}\right]^{T} \\
& =\left[\mathbf{E}_{3} \Phi\left(\delta_{1} T\right), \cdots, \mathbf{E}_{3} \Phi\left((p-1) T+\delta_{p} T\right)\right]^{T}
\end{aligned}
$$

Notice that $\Phi\left((i-j) T-\delta_{j} T\right)$ in (32) becomes a zero matrix when $j \geq i$.
Similarly, the analytical solution of the Poincaré map with $m_{y}=1$ for the current-mode dc drive system can be derived. Since the system always operates from stage 1 to stage 2, the duty cycle $\delta=\delta_{o n}$ is defined. By comparing (18) and (21), it shows that the differences are the replacement of all $\mathbf{E}_{\text {on }}$ by $\mathbf{E}_{\text {off }}$ and vice versa. Thus, the analytical modeling of the subharmonics derived previously is in fact a unified one for both voltage-mode and current-mode control. By setting $v_{r}(t)=0 \quad\left(v_{u}=v_{l}=0\right)$ and exchanging $\mathbf{E}_{\text {off }}$ and $\mathbf{E}_{o n}$ in the modeling of the voltage-mode controlled dc drive system, the analytical modeling of the current-mode controlled one can be obtained.

\section{COMPUTER SIMULATION}

To illustrate the derived modeling, computer simulation is carried out. The component and parameter values of the voltage-rnode $\mathrm{dc}$ drive system are as follows: $v_{l}=0 \mathrm{~V}$, $v_{u}=2.2 \mathrm{~V}, \quad T=4 \mathrm{~ms}, \quad g=2, \quad V_{i n}=100 \mathrm{~V}, \quad R=3.5 \Omega, \quad L=36 \mathrm{mH}$, $K_{E}=0.1356 \mathrm{~V} / \mathrm{rads}^{-1}, K_{T}=0.1324 \mathrm{Nm} / \mathrm{A}, B=0.000564 \mathrm{Nm} / \mathrm{rads}^{-1}$, $J=0.000971 \mathrm{Nm} / \mathrm{rads}^{-2}, T_{F}=0.39 \mathrm{Nm}, \omega_{\text {ref }}=100 \mathrm{rads}^{-1}$. Those of the current-mode dc drive system are as follows: $T=4 \mathrm{~ms}$, $V_{i n}=100 \mathrm{~V}, \quad g_{i}=1, \quad g_{\omega}=2, \quad g=2, \quad R=3.5 \Omega, \quad L=66 \mathrm{mH}$, $K_{E}=0.1356 \mathrm{~V} / \mathrm{rads}^{-1}, K_{T}=0.1324 \mathrm{Nm} / \mathrm{A}, B=0.000164 \mathrm{Nm} / \mathrm{rads}^{-1}$, $J=0.000571 \mathrm{Nm} / \mathrm{rads}^{-2}, T_{l}=0.39 \mathrm{Nm}, \omega_{r e f}=100 \mathrm{rads}^{-1}$.

\section{A. Bifurcation diagrams Using Numerical Computation}

By employing standard numerical techniques such as the brute-force algorithm [10] to compute the Poincaré map given by (12)-(15) for the voltage-mode dc drive system, the bifurcation diagrams of motor speed $\omega$ versus input voltage $V_{i n}$ and gain $g$ can be resulted as shown in Figs. 5 and 6, respectively. The corresponding bifurcation diagrams $\omega$ versus $V_{\text {in }}$ and $g$ by computing the Poincaré map given by (16) and (17) for the current-mode dc drive system are also shown in Figs. 7 and 8.

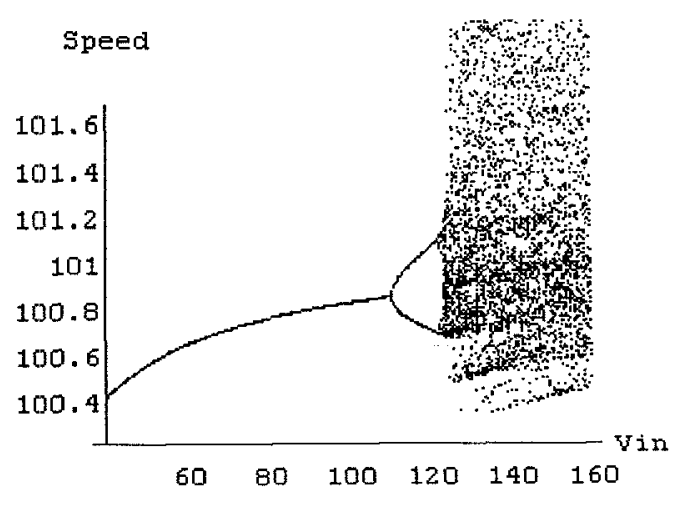

Fig. 5. Bifurcation diagram of $\omega$ vs $V_{\text {in }}$ using voltage-mode control. 


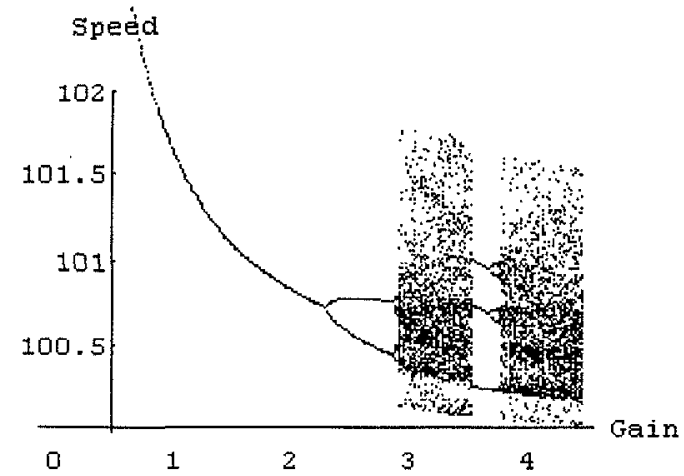

Fig. 6. Bifurcation diagram of $\omega$ versus $g$ using voltage-mode control.

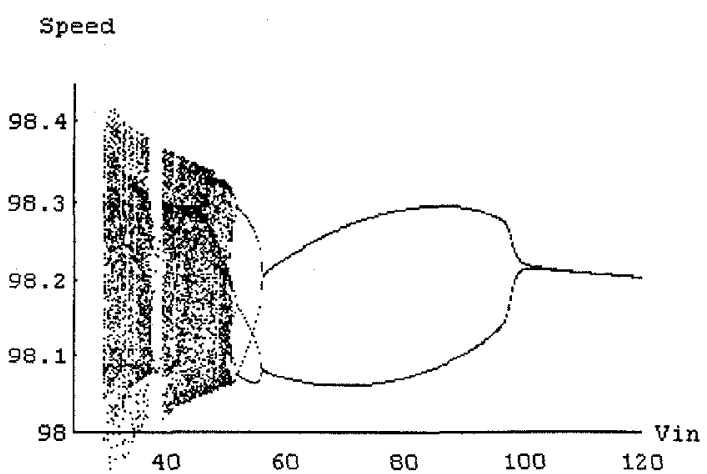

Fig. 7. Bifurcation diagram of $\omega$ vs $V_{\text {in }}$ using current-mode control.

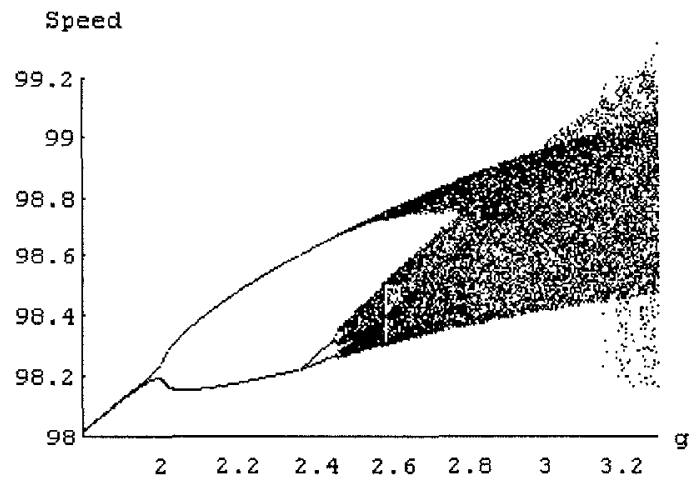

Fig. 8. Bifurcation diagram of $\omega$ versus $g$ using current-mode control.

It can be found that the system exhibits a typical perioddoubling route to chaos. As shown in Fig. 5, the voltagemode de drive system bifurcates to subharmonics and finally route to chaos as $V_{\text {in }}$ successively increases. On the contrary, when $V_{\text {in }}$ gradually decreases as shown in Fig. 7, the currentmode system emanates a period-doubling route to chaos. It indicates that the subharmonics and chaotic behavior caused by the same system parameter may be contrary when the drive system adopts different feedback control modes.

\section{B. Bifurcation Diagrams Using Analytical Approach}

Firstly, the voltage-mode dc drive system is analyzed. Based on the unified modeling in (22) for the period-1 orbit, the solution of duty ratio $\delta$ for a range of $V_{i n}$ varying from $40 \mathrm{~V}$ to $160 \mathrm{~V}$ is shown in Fig. 9. Hence, by using (23) and (24), the corresponding eigenvalues of the Jacobian matrix $D P$ is shown in Fig. 10 in which one of the amplitudes is greater than 1 when $V_{i n}>110.8 \mathrm{~V}$. It indicates that the period-1 orbit is unstable when $V_{i n}>110.8 \mathrm{~V}$.

Similarly, for the current-mode dc drive system, the duty ratio $\delta$ and the corresponding characteristic multipliers $\left|\lambda_{1}, \lambda_{2}\right|$ for $V_{\text {in }}$ varying from $30 \mathrm{~V}$ to $200 \mathrm{~V}$ is shown in Fig. 11. It can be found that one of the magnitudes is greater than 1 when $V_{\text {in }}<102 \mathrm{~V}$, indicating that the period-1 orbit is unstable when $V_{\text {in }}<102 \mathrm{~V}$.

It should be noted that the above analytical results closely agree with the previous numerical results. It is obvious that the required computational time based on the derived analytical solution is extremely less than that required for computation using the numerical brute-force algorithm. Thus, the proposed analytical approach can greatly facilitate the identification of the desired stable operating ranges of the $\mathrm{dc}$ motor drive system for different system parameters and conditions.

\section{PSpice Verification}

Based on the same operating parameters and conditions, realistic PSpice simulation of the drive system is performed. As shown in Fig. 12, the PSpice-simulated waveforms of $v_{\omega}$, $v_{i}$ and clock pulse at $V_{i n}=90 \mathrm{~V}$ illustrate that the system is operating at the period-2 orbit with $m_{i}=1$. When $V_{i n}=45 \mathrm{~V}$, those waveforms are in chaos as shown in Fig. 4. These system behaviors closely agree with the previous theoretical bifurcation diagrams.

\section{CONCLUSION}

In this paper, the second-order generalized iterative maps that describes the nonlinear dynamics of both voltage-mode and current-mode controlled dc drive systems during the continuous conduction mode of operation has been derived. Based on the derived map, computer simulation reveals that the system exhibits a typical period-doubling route to chaos. Moreover, the effect on subharmonics and chaotic behavior caused by the same system parameter may be contrary when the drive system adopts different feedback control modes. The unified analytical modeling for analyzing subharmonics of both voltage-mode and current-mode dc drive systems as well as their stability analysis based on the evaluation of the characteristic multipliers have also been presented. These can greatly facilitate the identification of the desired stable operating ranges with different system parameters and conditions. The theoretical results have been verified by comparing with the bifurcation points occurred during PSpice simulation. 


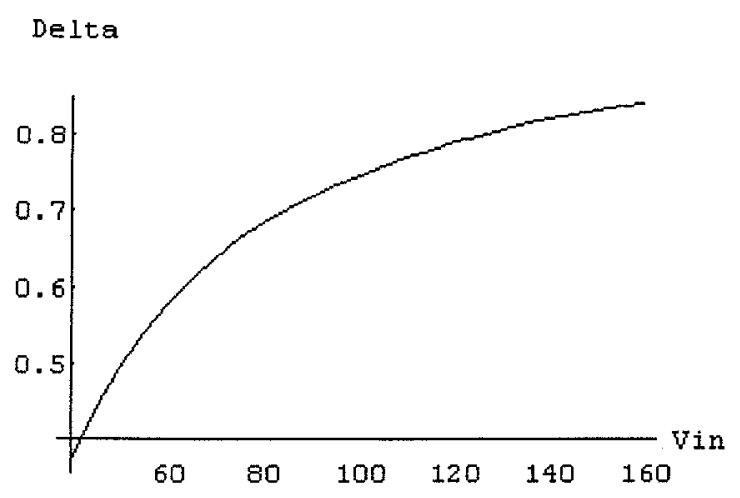

Fig. 9. Period-1 $\delta$ versus $V_{\text {in }}$ when voltage-mode control.

Eigenvalues

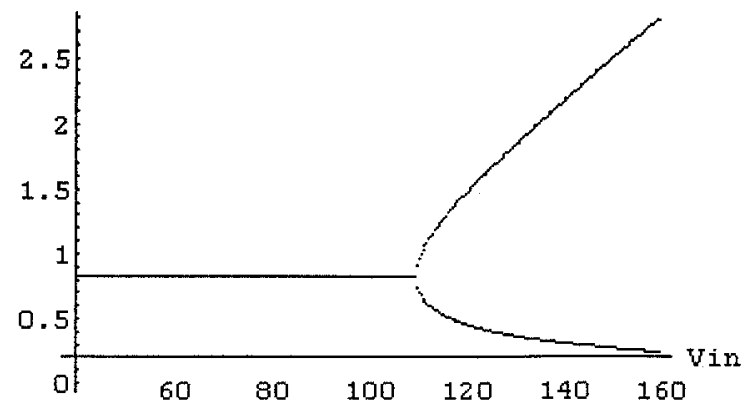

Fig. 10. Period-1 $\left|\lambda_{1}, \lambda_{2}\right|$ versus $V_{i n}$ when voltage-mode control.

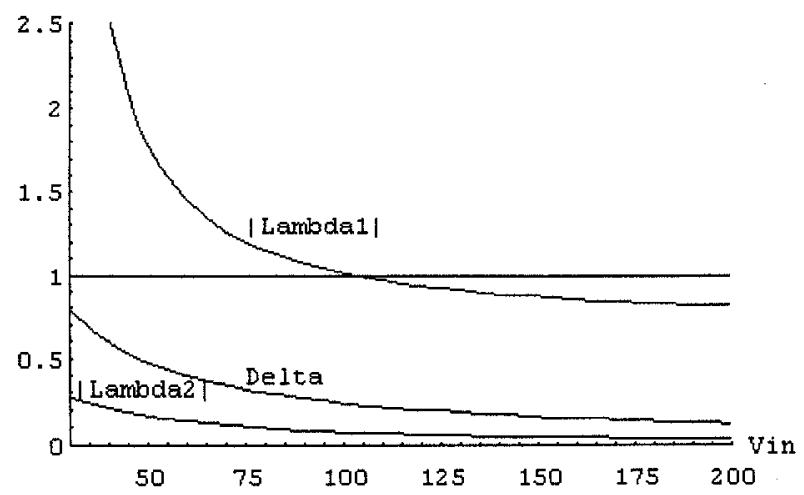

Fig. 11. Period-1 $\delta$ and $\left|\lambda_{1}, \lambda_{2}\right|$ versus $V_{i m}$ when current-mode control.

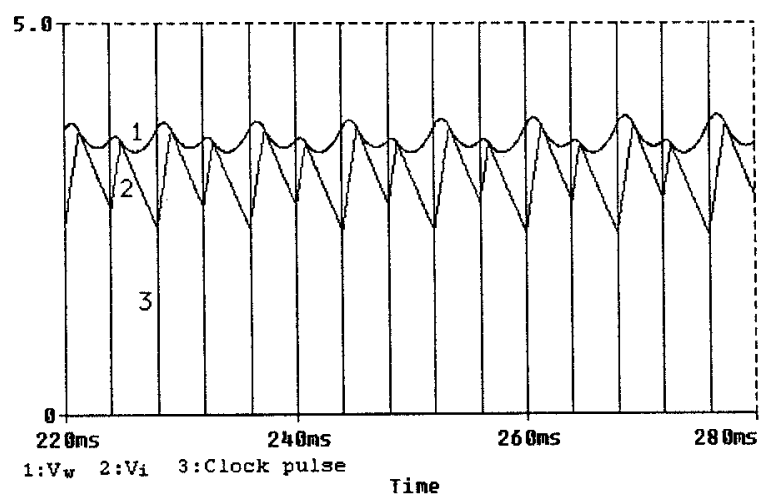

Fig. 12. PSpice-simulated $v_{\omega}, v_{i}$ and clock pulse for period-2 orbit.

\section{ACKNOWLEDGMENT}

This work was supported and funded in part by the Committee on Research and Conference Grants, the University of Hong Kong.

\section{REFERENCES}

[1] D.C. Hamill and D.J. Jefferies, "Subharmonics and chaos in a controlled switched-mode power converter," IEEE Transactions on Circuits and Systems, vol. 35, August 1988, pp. 1059-1061.

[2] J.H.B. Deane and D.C. Hamill, "Chaotic behavior in current-mode controlled dc-dc converter," Electronics Letters, vol. 27, 1991, pp. 1172-1173.

[3] D.C. Hamill, J.H.B. Dean, and D.J. Jefferies, "Modeling of chaotic DC-DC converters by iterated nonlinear mappings," IEEE Transactions on Power Electronics, vol. 7, January 1992 , pp. 25-36.

[4] J.H.B. Deane, "Chaos in a current-mode controlled boost dc-dc converter," IEEE Transactions on Circuits and Systems - I: Fundamental Theory and Applications, vol. 39, 1992, pp. 680-683.

[5] E. Fossas and G. Olivar, "Study of chaos in the buck converter," IEEE Transactions on Circuits and SystemsI: Fundamental Theory and Applications, Vol. 43, January 1996, pp. 13-25.

[6] I. Nagy, L. Matakas Jr. and E. Masada, "Application of the theory of chaos in PWM technique of induction motors," In Proceedings of International Power Electronics Conference, Yokohama, 1995, pp. 58-63.

[7] N. Hemati, "Strange attractors in brushless DC motors," IEEE Transactions on Circuits and Systems-I: Fundamental Theory and Applications, Vol. 41, January 1994, pp.40-45.

[8] K.T. Chau, J.H. Chen, C.C. Chan, J.K.H. Pong, and D.T.W. Chan, "Chaotic behavior in a simple dc drive," In Proceedings of IEEE Power Electronics and Drive Systems, Singapore, 1997, pp. 473-479.

[9] K.T. Chau, J.H. Chen, and C.C. Chan, "Dynamic bifurcation in dc drives," In Proceedings of IEEE Power Electronics Specialists Conference, St. Louis, USA, 1997, pp. 1330-1336.

[10] T.S. Parker and L.O. Chua, Practical Numerical Algorithm for Chaotic Systems. New York; SpringerVerlag, 1989. 